Colloque C2, supp1. au Journal de Physique II, Vol. 1, septembre 1991

\title{
CVD EXHAUST - SAFETY AND ENVIRONMENTAL SANITY
}

\author{
M.L. HAMMOND \\ PlasmaChem Inc, 505 East Evelyn Avenue, Mountain View, \\ CA 94041, U.S.A
}

\begin{abstract}
Both atmospheric pressure and low pressure CVD reactors generate substantial quantities of hazardous waste. Burn boxes and wet scrubbers remove much of this waste from atmospheric pressure exhausts; however, these methods are inefficient for CVD reactors using vacuum. Unless otherwise treated, the hazardous and toxic effluents distributed along the reactor exhaust system cause excessive vacuum pump maintenance, clogged exhaust lines, unscheduled downtime, the possibility of fire or other health/safety risks, and maximize the total cost of disposal. Scrubbing these effluents in the vacuum system before they reach the vacuum pump provides the safest and lowest cost method of handiling such toxic wastes.
\end{abstract}

\section{Introduction}

When vacuum pumps are required to process the corrosive, pyrophoric, and poisonous reactive gases required for deposition, the result is increased concerns for safety and maintenance costs, and a significant flow of toxic waste.

The shift to vacuum processing for CVD since 1975 has created new requirements for gas scrubbing to address the safety, maintenance cost, and toxic waste issues.

Safety issues requiring specific action include fire hazards, oil contaminated with poisonous hydride gases, potentially explosive materials in cold traps, and disposal of a large quantity of pump oil contaminated with a wide range of hazardous waste. 
Maintenance costs include the cost of oil and filters, unscheduled downtime, and the amount of required, hazardous, and labor-intensive repairs. Vacuum processing enhanced the productivity of CVD; however, the cost in terms of pump maintenance and clogged exhaust lines and ducts has been largely ignored.

oil-sealed pumps serving vacuum processors that handle reactive gas:

- Require changes of oil and filters one to four times per month or more with a loss of one to two hours each time

- Require repairs of pump and exhaust line one to five times per year with a loss of one or more shifts with each event

- Lose $5-40 \%$ of the oil into the house duct system

- Lose 15-25\% of the oil with each recycle

More recently, the semiconductor industry has turned to dry pumps in order to avoid the problems associated with oil pumps. Dry pumps eliminate the need for frequent oil changes; however, waste material accumulates within the pump and can force major rebuilds every 6 to 24 months. High flowrates of N2 $(30-50 \mathrm{~s} 1 \mathrm{~m})$ can minimize this accumulation, but the problem is only moved from the pump housing to the pump exhaust lines and the house ducts.

\section{CVD Vacuum Processor Effluent}

CVD reactors typically utilize only 10-15\% of the incoming gases in the vacuum processor. Therefore, exhaust lines require frequent cleaning of toxic, and sometimes pyxophoric, deposits, and vacuum pumps usually require excessive maintenance.

Gases that pass through the pump exhaust line must be scrubbed by water or by burning before the reaction products are removed in the house exhaust ducting. Sometimes, these gases are simply diluted with high flowrates of purge N2.

Gases that exit the vacuum pump often react in and clog the oil mist recovery systems. In order to maintain process uptime, the recovery systems are sometimes removed, leading to excessive loss of oil and further contamination of house ducts.

The toxic waste produced by vacuum processing includes not only the by-products of the reactive gases, but also the oil and filters. Further, this toxic waste flow includes the discarded pump parts and replaced exhaust lines and ducts. 
The matter of safety is even more important than the maintenance costs and toxic waste issues. Silane is a commonly used reactant for the deposition of polycrystalline silicon and silicon dioxide. Silane is pyrophoric in contact with air over a wide concentration range and has been the cause of several fires in semiconductor factories.

Silicon dioxide is often doped with boron or phosphorous using dopant hydrides which are poisonous at levels of only a few parts-per-milition. These dopants can be absorbed in the pump oil and in the deposits of the exhaust plumbing, and they can be released to the atmosphere upon exposure to water vapor in the air.

Proper handling of effluent from CVD and PECVD systems is essential for safety considerations as well as for minimum operations costs.

\section{Quantity of Toxic Waste}

The amount of toxic waste from a CVD process is surprisingly large. A reactor depositing Si3N4 with the SiH2Cl2 + NH3 reaction can produce up to 2190 lbs of solid material per year! This figure is based on a 300 sccm SiH2Cl2 flowrate with excess NH3, flowing $8000 \mathrm{hr} /$ year (91\% time utilization).

An SiO2 reactor using SiH4 and 02 (or other oxygen source) can produce up to 849 lbs of solids per reactor per year.

The TEOS/02 reaction will produce even more solids. At $3 \mathrm{ml} / \mathrm{min}$, up to $3168 \mathrm{lbs} / \mathrm{yr}$ of TEOS will be combined with 02 to produce 912 $\mathrm{lbs} /$ year of SiO2 and more than $2260 \mathrm{lbs}$ of various organic compounds. Depending upon the process conditions, most of the organic reaction products will be solids or liquids. Assuming only $80 \%$ of the organic products are condensable, the TEOS/O2 reaction can produce up to $1808+912=27201 \mathrm{bs} /$ year of solid material, most of which is waste.

Calculations of maximum solids production for these typical processes are summarized in Table 1 .

A ton of solid waste per reactor per year, as calculated here for the silicon nitride reaction, is a maximum value. More typically, time utilization of capital equipment is well below 91\%; however, even with a $20 \%$ time utilization, a nitride CVD process using $300 \mathrm{sccm}$ of SiH2Cl2 and excess NH3 will produce 460 lbs of solid waste per year or about $4.9 \mathrm{cu} . \mathrm{ft} / \mathrm{year}$. Even with only $100 \mathrm{sccm}$ of SiH2Cl2 and a $20 \%$ time utilization, the process will still produce more than $1.5 \mathrm{cu}$.ft. of solid waste per year. 
When this solid material is mixed in with the oil and oil filters, the total solid waste is increased by 300 to $500 \%$. More solid waste is generated if the surviving reactants are removed in a water scrubber, and even more waste is created when contaminated exhaust lines and ducts are removed from service.

\section{Distribution of Solid Waste}

Utilization of reaction product in a CVD reactor is quite small. Assuming a wafer throughput of $100150 \mathrm{~mm}$ wafers/hr with deposition on both sides and on the reactor walls, then the solid products of the CVD reactions for SiO2 and Si3N4 will be distributed approximately as outlined in Table 2. Less than $10 \%$ of the solids will be deposited on the wafers or on the walls of the reactor.

Distribution of solid waste within a vacuum processor depends strongly on the nature of the reactants. Precipitation of solid matter usually occurs where there is a sharp decrease in velocity or temperature. This observation has led to the occasional use of cold traps within the vacuum system. This practice has considerable risk in that the solid materials so trapped may react vigorously and possibly explode when exposed to air. Sometimes cold traps are allowed to warm up before opening them up to air. This action results the transfer a large amount of condensed material into the pump system at one time, causing even more service problems.

If the vacuum processor is equipped with an in-situ plasma or gaseous chemical cleaning capability, then the material deposited on the reactor wall will also be added to the solid waste in the exhaust system.

The cost and inconvenience of cleaning and replacing exhaust lines, processing many gallons of oil and attendant oil filters, and consuming unmeasured gallons of scrubbing water is generally not accounted for in the cost of operating a vacuum processor. 


\section{Solutions and Consequences}

To date, the industry's solutions to the problem of vacuum system effluent have been increased maintenance effort, use of high-cost perfluorcarbon (PFC) pump oils, exhaust burn boxes, and, most recently, dry vacuum pumps. All these techniques, by themselves, are inadequate for the task, expensive, and typically only change the nature of the hazardous waste problem without solving it.

Passive solutions such as filters and traps have high maintenance costs and do not solve the problems of fire hazard, handling toxic solids and gases, or unscheduled downtime. In addition, there is a risk that they will plug up and leave the vacuum processor full of a pyrophoric gas such as siH4 with no safe means to evacuate the system.

The use of PFC oils extends the life of the pump oil somewhat because PFC oil is less likely to degrade through chemical reaction or heat. Use of PFC oil is a partial solution because PFC oil is easily recycled. Unfortunately, each recycle results in a loss of $15-25 \%$ of the expensive oil.

Cost-effective use of $\mathrm{PFC}$ oil requires extending the time between oil changes. Scrubbing or removing the reactive gases before they reach the vacuum pump can extend the time between oil changes by preventing accuralation of reaction products in the pump and pump oil.

Dry pumps only move the problem to the pump exhaust line. When the pump exhaust lines are full, a shutdown is still required to clean up the problem. In addition, the required rebuilds and high $\mathrm{N} 2$ purge flowrates add significantly to the cost of operation.

Scrubbing reactive gases after they leave the pump meets the immediate safety requirements; however, this approach does nothing for the accumulation of hazardous materials in the vacuum exhaust line or the pumps. Failure to scrub the reactive gases before they enter the house duct system can lead to fire or, at a minimum, a clogged duct system.

Scrubbing reactive and toxic gases within the vacuum system before they reach the vacuum pump makes sense in terms of safety, economics, and environmental viewpoints because, with appropriate technology, these gases can be converted into relatively benign solids and gases while the reactants are still in their most concentrated form. This new technology is termed vacuum gas scrubbing. 


\section{Vacuum Gas Scrubbers}

Vacuum gas scrubbers use high intensity plasma energy to complete the reactions already started in the process chamber. Such vacuum gas scrubbers are able to operate within the vacuum because, for most CVD processes, pressures are within the range of easy plasma generation ( 0.1 to 5 torr). Safety issues are addressed because most reaction products are generally less hazardous than the reactants.

As a note of caution, not all reactant gases lose their toxicity in a vacuum gas scrubber. For example: AsH3 maintains its poisonous nature even as an oxide, and $\mathrm{H} 2$ retains its flammability until oxidized.

Vacuum gas scrubbing reduces the total amount of toxic gases in the exhaust stream, prevents build-up of solid waste in the vacuum pump and in the downstream exhaust lines, and reduces the total amount of waste material to be handled.

When a vacuum gas scrubber is placed downstream from a throttle valve, the chemical and physical reactions occurring there will have no effect on the pressure or reactions in the process chamber. Even without a throttle valve, it is relatively simple to design an exhaust system in which backflow is eliminated.

Vacuum gas scrubbers offer the additional advantage of reacting any pump oil vapor that reaches the scrubber chamber before the vapor migrates to the process chamber. This capability provides the same advantage claimed by dry pumps; namely, elimination of of oil backflow. It should be noted that appropriate purge flows and valving will also prevent oil backflow.

\section{Scrubbing Efficiency}

Scrubbing efficiencies vary with different processes and with different scrubber designs. Removal efficiencies of greater than 90\% have been achieved for most typical CVD reactants and pressures.

cost reduction is another reason to consider vacuum gas scrubbing. Maintenance costs of vacuum processing range from $\$ 30,000$ to $\$ 50,000$ per year per system or more depending upon the process. This cost includes depreciation on the pumps and scrubbers plus the costs of oil and filter changes, clean/repair of pumps, clean/repair of exhaust lines and ducts, and maintenance of downstream scrubbers. 
By removing $90 \%$ of the reactants before they reach the pump, the time between required pump maintenance activities will increase by at least 2-5x. Other sources of oil deterioration may prevent the time between oil changes to be extended by 10x. The use of a vacuum gas scrubber reduces the cost in every comparison, and makes the cost of using PFC oil comparable to that for using hydrocarbon oil.

8. The Future

Three key concerns in manufacturing for the 1990s are:

- Safety

- Environmental impact

- Operating costs

A competitive economy forces efficient manufacturing operations, and vacuum gas scrubbers provide one means to reduce total operating costs.

Concern for the environment has focused considerable attention on the total amount of hazardous material being handled and disposed of by semiconductor manufacturing and by other industries. As the volume of devices increases each year, the semiconductor industry will be forced to be more efficient in terms of chips produced per unit volume of hazardous waste. Vacuum gas scrubbing offers one answer to that problem.

Safety must be an overriding concern in all high technology manufacturing, especially in the maintenance and operating areas where personnel have not had the educational background to fully understand the chemistry and physics involved in various processes.

In comparison to the manner in which effluent from vacuum processors is being handled today, vacuum gas scrubbing provides economic safety and environmental sanity. 


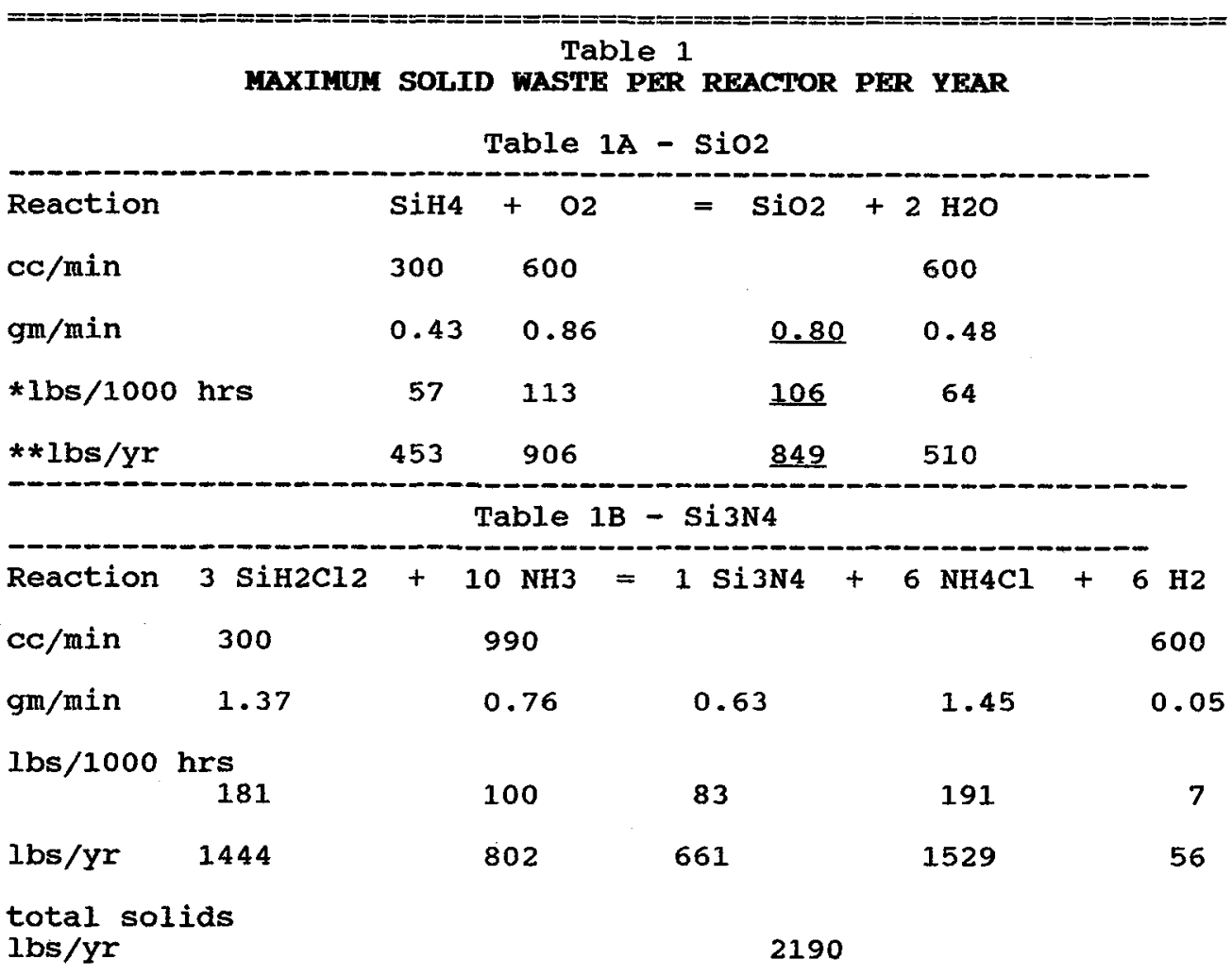

Table 1C - TEOS SiO2

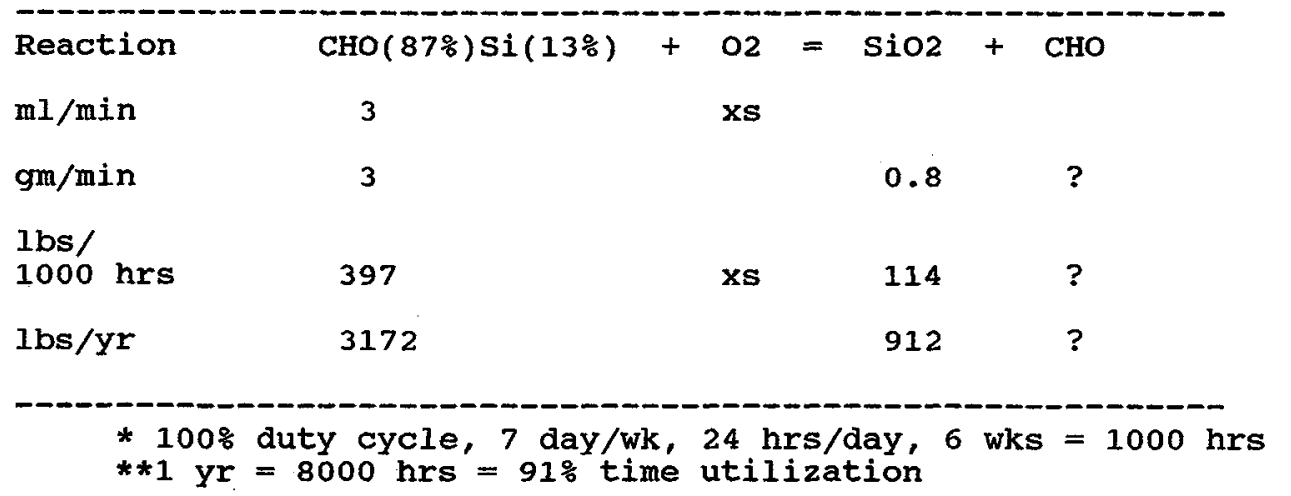




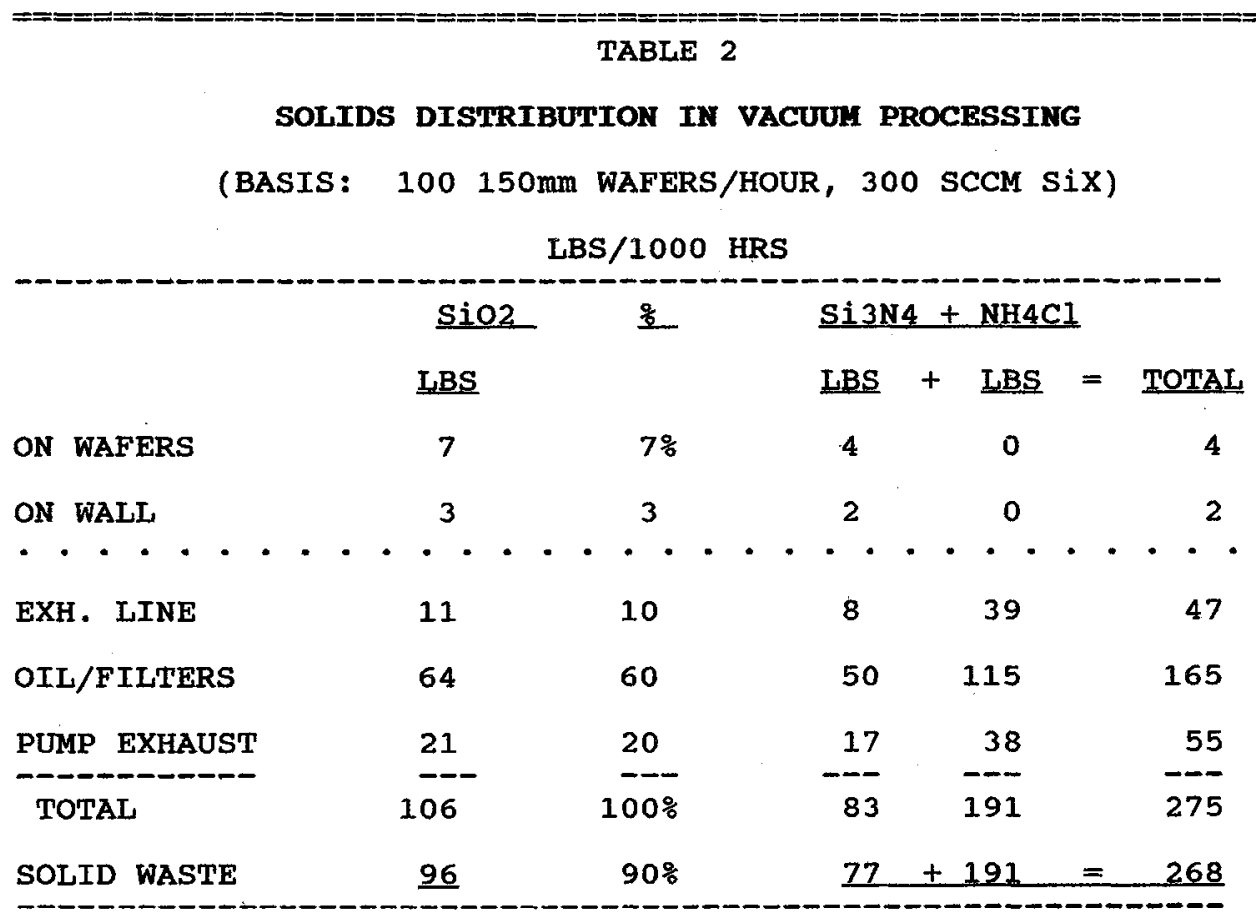

LBS/8000 HRS

SOLID WASTE $\quad \underline{768}+1528=2144$

